\title{
Polyvinyl Alcohol Based Nanocomposite Films Engineered With Clove Oil and Chitosan Nanoparticles to Combat the Postharvest Fungal Diseases
}

Radhakrishnan EK ( $\nabla$ radhakrishnanek@mgu.ac.in )

Mahatma Gandhi University https://orcid.org/0000-0003-2688-701X

Ashitha Jose

Mahatma Gandhi University

\section{A.S Saranya}

St Thomas College, Pala, Kottayam, India

Chinnu Chacko

St Thomas College, Pala, Kottayam, India

Dhanya M Jacob

Mahatma Gandhi University

\section{Research Article}

Keywords: chitosan nanoparticle, clove oil, antifungal

Posted Date: February 25th, 2021

DOI: https://doi.org/10.21203/rs.3.rs-236333/v1

License: (c) (1) This work is licensed under a Creative Commons Attribution 4.0 International License.

Read Full License 


\section{Abstract}

The post-harvest loss of fresh produces is a global challenge to the food industry. The drastic loss of fresh produces caused mainly by fungal attack demands the development of active packaging materials with antimicrobial properties. Hence studies have already reported the applications of polymers like Polyvinyl Alcohol (PVA) engineered with antimicrobial properties for the packaging. In the current study, material properties of PVA alone, PVA incorporated with chitosan nanoparticles (PCS), clove oil (PCO) and also chitosan nanoparticles and clove oil combination (PCSCO) have been studied for the packaging applications. All the developed films were characterised by the XRD and FTIR analysis and it confirmed the molecular interactions among the components of nanocomposites. At the same time, the bionanocomposite PCSCO was found to have low moisture content and film solubility as suitable for the packaging application. In addition, the presence of chitosan nanoparticles and clove oil was found to provide the microbial barrier properties to the PCS, PCO and PCSCO flms. The PCSCO film with both chitosan nanoparticles and clove oil was further demonstrated to have superior antifungal activity against the spoilage organism Pythium aphanidermatum. The results of the study indicate the potential of developed nanocomposite for the postharvest packaging application.

\section{Introduction}

The environmental concern associated with the plastic pollution has led to a paradigm shift towards the replacement of the petro-chemical substances by the eco-friendly and biodegradable materials. For the same, various polymers have already been engineered for the tailor-made properties through polymer blending. The improved material properties of nanoparticle doped polymers have already been identified to have immense promises for the packaging applications [1]. Here, the distribution of dispersed phase nanoparticles in the polymer matrix makes the nanocomposites to have desirable properties which can further be improved through the introduction of antimicrobial components [2]. This is of significant importance in the food industry where the microbial degradation and oxidative damage seriously affect the shelf life and properties of the food material. Hence the active packaging, facilitated through the incorporated biological agents can improve the quality and safety of the packed food without compromising the material properties. For the same, various polymers have already been engineered with natural components to make the packaging material to have antimicrobial properties [3]. Among the various polymers used for the engineered packaging applications, poly vinyl alcohol (PVA) is an excellent choice due to its compatibility and desirable physico - chemical properties. PVA has also been exploited for the applications in biomedicine due to its higher water solubility, lower cytotoxicity and easy processing [4]. Its preference as a packaging material is also because of its excellent barrier properties against oxygen and carbon dioxide. Engineering the PVA with various nanoparticles has also been described to improve its material properties for tailor made applications [5].

In a previous study, the active packaging material developed by fabricating the PVA matrix with quaternary ammonium salt modified chitosan [6] and chitosan/ bentonite [7] has reported to have better antibacterial efficacy. As the bacterial contamination is a major issue affecting the quality of the packed 
food, the antibacterial packaging will have significant applications [8]. Chitosan blended PVA has been widely accepted in pharmaceutical and packaging industries owing to its non-toxic nature, biocompatibility, and ease of development and characterization [1]. The incorporated chitosan has also been reported to improve the thermal and physicochemical properties of the polymer matrix [9]. Moreover, the water insolubility of chitosan results in lower swelling index and permeability which is highly desirable for the packaging application [10]. In a study, chitosan nanoparticles have been described to be operative in reducing the water content of the prepared composite films along with its successful antibacterial efficacy [11].

Essential oils with broad-spectrum antibacterial properties have recently been widely exploited for the development of active packaging materials. Due to their multi-mechanistic effect against bacteria, these can be inhibitory to the resistant organisms also [12]. By incorporating the essential oil as the structural part of the packaging materials, its concentration can be kept below the organoleptically accepted values [13]. Among the essential oils, the clove oil has been documented under the GRAS (generally recognized as safe) category and has wider applications in packaging and medical sectors due to its broad spectrum antibacterial properties [14]. Clove oil has also been reported to be antifungal against the postharvest pathogens like L. theobromae and C. gloeosporioides [15]. Recently, it has been suggested to be effective in the preservation of frozen bluefin tuna [16] and smoked salmon [17]. Considering the antimicrobial efficacy of boththe chitosan nanoparticles and clove oil, the current study has been designed to develop PVA based nanocomposites by incorporating these components. From the results of the study, the developed nanocomposites were found to have the promises for packaging applications and preventive effect against the postharvest fungal pathogens.

\section{Materials And Methods}

\subsection{Materials}

Polyvinyl alcohol (PVA) (hot water soluble and fully hydrolysed, PCT1316-100G), with an average MW of 60,000-1,25,000 Da was purchased from HiMedia laboratories Pvt Ltd. (India). Clove oil was purchased from a local medical shop, chitosan from marine hydrocolloids, Ernakulam, acetic acid from Merck (Worli, Mumbai, India) and TPP from Sigma-Aldrich (238503).

\subsection{Preparation and characterization of chitosan nanoparticles}

\subsubsection{Preparation of chitosan nanoparticles}

Chitosan nanoparticles (CSNP) were synthesized according to the modified ionic gelation method [18]. Briefly, $0.9 \%$ of the chitosan solution was prepared in $1 \%(\mathrm{v} / \mathrm{v})$ acetic acid by continuous stirring (for 2-3 hours) at $65^{\circ} \mathrm{C}$. After obtaining a clear solution, $0.25 \%$ TPP solution was added (6:1 ratio of chitosan to TPP) drop wise while stirring and allowed the reaction to continue for another 30 minutes. The CSNPS formed were further stored at $4^{\circ} \mathrm{C}$ and used directly without any further purification. 


\subsubsection{Characterization of synthesized nanoparticles using DLS and TEM}

The particle size distribution and average size of synthesized chitosan nanoparticles were analysed using a particle size analyser (HORIBA nanopartica nanoparticle analyser SZ-100) with sample holder temperature of $25^{\circ} \mathrm{C}$ [19]. For further confirmation of the shape and size characteristics of the prepared nanoparticles, HR-TEM (JOEL 2100- HRTEM) analysis was carried out wherein, the samples were prepared on a carbon coated copper TEM grids [20].

\subsection{Development of bionanocomposite films}

Here, the PVA thin film and the modified films containing clove oil and chitosan nanoparticles were prepared by solvent casting technique with slight modifications [21]. The PVA control film was prepared by mixing $1.2 \mathrm{~g}$ of PVA in $40 \mathrm{~mL}$ distilled water with magnetic stirring at $65 \mathrm{C}$ until a clear solution was obtained. This was further casted onto the sterile Petri plate and dried to obtain the films. For the preparation of PVA film with clove oil (PCO), $0.4 \%(\mathrm{v} / \mathrm{v})$ clove oil was added into the clear PVA solution prepared as described above. This solution was then mixed for another 1 hour at $1000 \mathrm{rpm}$ at room temperature and further casted to obtain the PCO films. For the development of PVA film with chitosan nanoparticles (PCS), 3\% (w/v) PVA was dissolved in the already synthesized CS solution followed by mixing at $70 C$ until the PVA was completely dissolved. After the complete dissolution, the solution was casted onto sterile Petri plate and dried to obtain the film. The addition of $0.4 \%(\mathrm{v} / \mathrm{v})$ oil into the PCS solution prepared as described above was further processed to get the PCSCO film.

\subsection{Characterization of the developed bionanocomposites}

\subsubsection{Fourier transform infrared spectroscopy (FTIR) and X-ray diffraction (XRD) analysis}

Fourier transform infrared spectroscopy of the developed bionanocomposite was performed using Shimadzu IR Prestige 2 FTIR Spectroscopy in the attenuated total reflectance mode (ATR). The analysis was done in the scan range of $4000-500 \mathrm{~cm}^{-1}$ with a scan rate of $4 \mathrm{~cm}^{-1}$.

The X-ray diffraction pattern of the film samples was recorded using Xpert3 PANalytical X -ray diffractometer. All the samples were scanned in the continuous scan mode in the $2 \theta$ range of 20 to 80 . The machine was equipped with Nifiltered Cua radiation $\left(\lambda=1.54 \mathrm{~A}^{\circ}\right)$ under the conditions of $45 \mathrm{kV}$ and $30 \mathrm{~mA}$ at a step size of $2 \theta=0.02^{\circ}$.

\subsubsection{Estimation of the hydrodynamic properties of the prepared nanocomposite films}

For the analysis of the water holding capacity $(\mathrm{WHC})$ of the prepared films, small pieces of film samples $(2 \times 2 \mathrm{~cm})$ were cut in triplicate, weighed $\left(\mathrm{W}_{1}\right)$ and immersed in distilled water for 2 minutes, the final weight $\left(\mathrm{W}_{2}\right)$ of the samples were then noted after removing the excessive water by blotting with filter paper [22]. The water holding capacity of the film was calculated with the following equation, 
$\mathrm{WHC}=\frac{W_{2}-W 1}{W 1} * 100$

Similarly, for the moisture content analysis, small pieces of the films were cut $(3 \times 3 \mathrm{~cm})$ in triplicates and weighed $\left(W_{a}\right)$ and then dried at $105^{\circ} \mathrm{C}$ for 24 hours in hot air oven and the dry weight $\left(W_{b}\right)$ was recorded [23]. The moisture content (MC) was calculated based on the water removed from the films using the following equation,

$\mathrm{MC}=\frac{W a-W b}{W b} * 100$

\subsection{Microbial barrier potential of the developed bio nanocomposite films}

To analyse the barrier potential of the films, the mouth of the sterile McCartney tubes containing $5 \mathrm{~mL}$ of nutrient broth was covered aseptically with the developed films after the UV sterilization. Parafilm was used to seal the edges of the film. This setup in triplicate was then incubated under normal laboratory condition for 7 days. At the end of the incubation, the tubes were observed for the visible changes in turbidity. Then, $100 \mu \mathrm{L}$ of the culture medium from each of these vials was spread plated onto sterile nutrient agar medium and was incubated at room temperature for the confirmation of microbial growth.

\subsection{Antifungal Potential of the developed bionanocomposites}

The bionanocomposites were analysed for its antifungal potential against the pathogen Pythium aphanidermatum. For this, a piece of the UV sterilized thin film was placed aseptically on one half of the PDA plate, while the pathogen was inoculated on the other half and allowed to grow towards the film. The organism was allowed to grow for 3 days at $27^{\circ} \mathrm{C}$ and the growth pattern was noted.

\section{Results And Discussion}

\subsection{Characterization of chitosan nanoparticles}

\subsubsection{DLS and TEM}

The DLS analysis of the synthesized chitosan nanoparticles showed the average particle size distribution to be $3.1 \mathrm{~nm}$ with a polydispersity index of 1.863 . This indicated the broad range size distribution of the synthesized nanoparticles (Fig. 1).

The HR-TEM analysis revealed the chitosan nanoparticles to be well dispersed with a primary spherical shape and the size distribution was in unison with that of DLS analysis (less than $10 \mathrm{~nm}$ ) (Fig. 2). The morphological analysis result using HR-TEM is confirmatory to the shape and size of the synthesized nanoparticles [20].

\subsection{Appearance of the prepared bionanocomposites}


All the prepared films (P, PCO, PCS and PCOCS) were flexible and could be peeled off from the Petri plates with ease. The addition of chitosan nanoparticles was found to reduce the smoothness of the film surface considerably along with a slight reduction in the flexibility of the films. However, all the prepared films except the PVA control film had a slight yellow colour (Fig. 3).

\subsection{Characterization of the developed bionanocomposite films}

\subsubsection{FTIR and XRD analysis}

The possible interactions within the developed bionanocomposite films were analysed using the FTIR analysis. Here, a broad and intense peak was observed at $3600 \mathrm{~cm}^{-1}$ for all the developed bionanocomposite films which could be due to the characteristic hydroxyl group (OH) stretching band of both inter and intra molecule hydrogen bonds (Fig. 4). This hydroxyl group has previously been described to be responsible for the water holding capacity of the PVA hydrogels [24]. The broadening of the peak at $3600 \mathrm{~cm}^{-1}$ observed in the PCS film could be attributed to the hydrogen bonds formed due to the presence of acetic acid, which acted as the solvent in the chitosan nanoparticle synthesis. The intensification of these peaks and its broadening in different bionanocomposites indicated the interaction between the individual components especially in the PCSCO films. A peak observed at $2914 \mathrm{~cm}^{-1}$ in the P neat film could be characteristic of the asymmetric $\mathrm{C}-\mathrm{H}$ vibration caused by the saturated $\mathrm{C}-\mathrm{H}$ groups of methyl group $\left(\mathrm{CH}_{3}\right)$. Eventhough the peak was not evident in the PCO film, it was clear in the PCSCO with a high intensity. The $\mathrm{C}-\mathrm{H}$ wagging vibrations in the developed films might have resulted in the peak at $1421 \mathrm{~cm}^{-1}$. The shift in the band and changes in the intensity of the peak indicated the $\mathrm{CH}$ asymmetric stretching and it confirmed the interaction of individual components in the matrix.

The PCS film showed specific peaks which could be due to the presence of eugenol, the major component in clove oil. The peaks at 1271.09 and $1207.44 \mathrm{~cm}^{-1}$ could be attributed to the $\mathrm{C}=0$ groups of eugenol, while the peaks at 1606.7, 1514.12 and $1454.33 \mathrm{~cm}-1$ indicated the C-C interactions (stretching vibrations of aromatic $\mathrm{C}=\mathrm{C}$ ) of eugenol, eugenol acetate and methyl 2-hydroxybenzoate. The intense FTIR peaks in the region $1300-1000 \mathrm{~cm}^{-1}$ indicated the presence of $\mathrm{C}=0$ vibrations of the ether and alcohol functional groups of eugenol and eugenol acetate. The retention of these peaks in the PCSCO films confirmed the presence of the essential oil in the matrix along with the chitosan nanoparticles [25-27]. The shift observed in these peaks could be attributed to the interactions among the individual components of the bionanocomposite.

The band observed at $3419 \mathrm{~cm}^{-1}$ could be attributed to the combined peaks of the $\mathrm{NH} 2$ and $\mathrm{OH}$ stretching vibrations of chitosan. The peak at 1552 (gamma NH) was sharper than that at $1641 \mathrm{~cm}^{-1}$ which indicated the higher degree of deacetylation of the chitosan [28]. However, there were slight shift in the peaks in all the chitosan nanoparticle based films when compared with the pure chitosan nanoparticles which could be attributed to the interactions between the PVA matrix and the nanoparticles. The peaks observed between $1600-1800 \mathrm{~cm}^{-1}$ could be attributed to the $\mathrm{C}=0$ and $\mathrm{C}=\mathrm{C}$ bonds present in the major components of the clove oil like the eugenol, eugenyl acetate and caryophyllene [24]. Similarly, a number 
of smaller peaks were observed at the region $1600-600 \mathrm{~cm}^{-1}$ which could be the characteristic feature of $\mathrm{C}=\mathrm{O}$. However, when combined with the chitosan as in the case of the PCSCO film, the interactions with the constituents might have resulted in the reduced number of low intensity peaks. Based on the observed changes in FTIR width, intensity and shifting of peaks, the interaction among the constituents of the nanocomposite could be confirmed.

The mechanical properties of the polymer nanocomposite blend films could be influenced by its crystalline nature. The XRD analysis of the PVA neat film revealed strong crystalline reflection at $2 \theta$ value of 19.69 with a shoulder peak at 25.92 (Fig. 5). This represented the reflections (101 and 200) from a monoclinic unit cell [29]. These peaks were retained in all the prepared films with slight shift (19.76 and 25.902 for the PCO, 19.64 and 25.79 for the PCS, 19.71 and 25.96 for the PCSCO) which could be due to the interaction of PVA with the individual constituents. In the case of PCS film, a new peak could be observed at $2 \theta$ value 22.98 which might have originated due to the amorphous structure of the nanoparticle. However, the peak was not evident in the PCSCO film due to the interactions among the individual components. The broadening and intensification of the peaks in the PCSCO film further indicated the higher amorphous nature of the nanocomposite film attributed to the presence of chitosan nanoparticle [30].

\subsubsection{Estimation of the hydrodynamic properties of the prepared nanoacomposite films}

Modified atmospheric packaging has been accepted widely to enhance the shelf life of the packed food[31]. Hence, the hydrodynamic potential of the packaging material can have determining effect on the shelflife of the food. For the packaging material with the enhanced water affinity, there will be high possibility for the food material to get damaged due to the contact with water [32]. In the current study, the moisture content of the P neat film $(39.63 \pm 4.75)$ and PCS films $(41.04 \pm 1.47)$ were found to be comparatively high when compared with the PCO (17.915 \pm 1.15$)$ and PCSCO (18.8 \pm 3.36$)$ films (Fig. 6). This might be due to the hydrophobic nature of the $\mathrm{CO}$ present in these films. From the results of the study, the incorporation of $\mathrm{CO}$ can be confirmed to improve the moisture content of the native $\mathrm{P}$ film and thereby favouring its suitability as a promising packaging material. Here, the hydrophobic nature of the active components could be considered to affect the sorption kinetics of the prepared films negatively. The addition of neem oil has previously been described to lower the moisture content of the polymerbased film and thereby enhancing its packaging efficacy. The differential behaviour of the oil containing film has also been shown to be related to the specific interactions between the oil components and matrix in a concentration dependent manner [33]. As the presence of moisture is found to increase the $\mathrm{O}_{2}$ permeability of the films, the ethylene-vinyl copolymers including polyamides and poly vinyl alcohol with high moisture sorption properties have limited applications under high-moisture conditions of packaging [34][35]. Hence an ideal packaging material should have lower film solubility to prevent the deterioration of the packed food material [36].

In the study, the solubility of the P neat film $(19.115 \pm 1.71)$ was found to be reduced with the addition of clove oil as in the case of PCO film (10.4 \pm 2.03$)$. On the contrary, a two-fold increase in solubility could be 
observed for the PCS films (43.74 \pm 2.03$)$ (Fig. 7). This hike was further observed to get reduced in the PCSCO films $(25.7 \pm 2.0)$ due to the interaction of individual components. With the higher number of cationic groups present in chitosan nanoparticles, there will be better repulsion between the chains and the solvation with water molecules here can lead to the increased swelling degree of the prepared films. The concentration of nanoparticles used for the film formation can also have influential impact on tortuous pathway creation and thereby the hydrodynamic potential of the developed films. However, use of high concentration of nano chitosan for the film preparation is considered to render its surface rough and thick resulting in the formation of numerous large holes in the film matrix. This can ultimately increase the water vapour transmission rate and the solubility [37]. From the results of the study, these changes are observed to get modified with the incorporation of essential oils and thereby making the film ideal for packaging applications.

\subsection{Microbial barrier property of the prepared films}

Prevention of entry of microorganisms is a desirable quality for the packaging material[38]. Hence, microbial barrier property of the films prepared in the study was conducted (Fig. 8). Upon the visual analysis, the bottles covered with PVA control films showed enhanced turbidity when compared with the tubes covered using PCS, PCO and PCSCO films. This was further confirmed by the affluent microbial growth on nutrient agar plates for the samples collected from PVA control film covered bottles. However, no microbial growth was observed for samples from other bottles and the result was in accordance with the visual acuity. Here, all the bionanocomposites with the incorporated clove oil or chitosan nanoparticles or their combination were found to have the ability to prevent microbial penetration and thereby favouring its application as promising packaging material. However, the PCSCO film, which contained both the chitosan nanoparticles and clove oil was observed to have superior barrier property than other films. This could be due to the synergistic activity of the clove oil and chitosan nanoparticles used for the film preparation. Thus, the bionanocomposite films developed in the study can have the promises to enhance the shelf life of the packed food by preventing the entry of external microbial agents.

\subsection{Antifungal properties of the developed bionanocomposites}

Pythium aphanidermatum is a pathogenic fungus that can infect fruits and vegetables[39,40]. Hence, the antifungal property of the developed bionanocomposites was analyzed against the Pythium spp. From the results, the PVA control film was found not to possess any antifungal property. But the PCS and PCO films were found to be inhibitory to the fungal growth. Here also superior inhibition could be observed for the PCSCO film (Fig.9). This could be attributed to the synergistic effect of clove oil and chitosan nanoparticles. Chitosan in its bulk and nanoparticle forms have already been described to have activity against the $P$. aphanidermatum [41]. Similarly, the clove oil is also known for its wide range of antibacterial, antiviral and antifungal activities due to the presence of compounds such as carvacrol, thymol, eugenol and cinnamaldehyde [42]. Clove oil has also been described to be antifungal against Fusarium and Rhizoctonia through induction of conidial malformation and disruption of the fungal 
growth [43]. The chitosan nanoparticles and essential oils when used in combination have already proven to be highly effective against the fungal pathogens [44]. Hence, these combinations and the films prepared from these will have significant promises to limit the use of synthetic fungicides and can thus help to alleviate the deleterious environmental impact of the toxic chemicals. Moreover, the packaging films incorporated with these will be safe to use as the individual components are coming under the GRAS category. Thus, the developed active antifungal packaging films will have applications to minimise the post-harvest loss of the fresh produces.

\section{Conclusion}

Post-harvest loss caused by the microorganisms is a major agricultural issue. The recent trends in packaging sector focus mainly to develop active packaging materials with inherent antimicrobial and improved barrier properties. Hence in the current study, PVA based bionanocomposites were fabricated with chitosan nanoparticles and clove oil to explore their applications in packaging. The chitosan nanoparticles prepared in the study were characterized by DLS and TEM and the bionanocomposites were characterised by using FTIR and XRD. From the results of the study, the PCSCO film containing both chitosan nanoparticle and clove oil was found to have the supreme potential as antimicrobial packaging material to ensure the food safety by enhancing the shelf life and preventing the postharvest loss.

\section{Declarations}

\section{Acknowledgments}

We are highly thankful to Kerala State Plan Fund for research grant of a Project entitled "Exploring Indigenous Microbiome of Kerala for Natural and Organic Agricultural Practices - an Approach to Green Kerala". Authors also acknowledge KSCSTE-KBC-YIPB and JAIVAM project for the support.

\section{Conflict of Interest}

The authors declare that they have no conflict of interest.

\section{References}

1. M. J. Tommalieh, H. A. Ibrahium, N. S. Awwad, and A. A. Menazea, J. Mol. Struct. 1221, 128814 (2020).

2. R. Sharma, S. M. Jafari, and S. Sharma, Food Control 112, 107086 (2020).

3. G. Pinheiro Bruni, J. P. de Oliveira, L. G. Gómez-Mascaraque, M. J. Fabra, V. Guimarães Martins, E. da R. Zavareze, and A. López-Rubio, Food Packag. Shelf Life 23, 100426 (2020).

4. R. Bryaskova, D. Pencheva, G. M. Kale, U. Lad, and T. Kantardjiev, J. Colloid Interface Sci. 349, 77 (2010). 
5. M. Zamanian, H. Sadrnia, M. Khojastehpour, F. Hosseini, B. Kruczek, and J. Thibault, J. Polym. Environ. (2020).

6. T. Min, Z. Zhu, X. Sun, Z. Yuan, J. Zha, and Y. Wen, Food Chem. 308, 125682 (2020).

7. M. Koosha and S. Hamedi, Prog. Org. Coatings 127, 338 (2019).

8. T. Min, X. Sun, Z. Yuan, L. Zhou, X. Jiao, J. Zha, Z. Zhu, and Y. Wen, LWT 135, 110034 (2021).

9. M. Hamdi, R. Nasri, S. Li, and M. Nasri, Food Hydrocoll. 89, 802 (2019).

10. O. F. Nwabor, S. Singh, S. Paosen, K. Vongkamjan, and S. P. Voravuthikunchai, Food Biosci. 36, 100609 (2020).

11. S. Ju, F. Zhang, J. Duan, and J. Jiang, Carbohydr. Polym. 237, 116167 (2020).

12. S. Purkait, A. Bhattacharya, A. Bag, and R. R. Chattopadhyay, Arch. Microbiol. 202, 1439 (2020).

13. S. R. Muppalla, S. R. Kanatt, S. P. Chawla, and A. Sharma, Food Packag. Shelf Life 2, 51 (2014).

14. I. Unalan, S. J. Endlein, B. Slavik, A. Buettner, W. H. Goldmann, R. Detsch, and A. R. Boccaccini, Pharmaceutics 11, 570 (2019).

15. G. Nandhavathy, V. Dharini, P. Anand Babu, R. B. Nambiar, S. Periyar Selvam, E. R. Sadiku, and M. Mahesh Kumar, Mater. Today Proc. (2020).

16. I. Echeverría, M. E. López-Caballero, M. C. Gómez-Guillén, A. N. Mauri, and M. P. Montero, Int. J. Food Microbiol. 266, 142 (2018).

17. N.-B. Song, J.-H. Lee, M. Al Mijan, and K. Bin Song, LWT - Food Sci. Technol. 57, 453 (2014).

18. T. V. Nguyen, T. T. H. Nguyen, S.-L. Wang, T. P. K. Vo, and A. D. Nguyen, Res. Chem. Intermed. 43, 3527 (2017).

19. M. Bodnar, J. F. Hartmann, and J. Borbely, Biomacromolecules 6, 2521 (2005).

20. R. Thomas, S. Snigdha, K. B. Bhavitha, S. Babu, A. Ajith, and E. K. Radhakrishnan, 3 Biotech 8, 404 (2018).

21. S. Mathew, A. Jayakumar, V. P. Kumar, J. Mathew, and E. K. Radhakrishnan, Int. J. Biol. Macromol. 139, 475 (2019).

22. S. Mathew, S. Snigdha, J. Mathew, and E. K. Radhakrishnan, Appl. Clay Sci. 161, 464 (2018).

23. I. dos Santos Paglione, M. V. Galindo, J. A. S. de Medeiros, F. Yamashita, I. D. Alvim, C. R. Ferreira Grosso, L. S. Sakanaka, and M. A. Shirai, Food Packag. Shelf Life 22, 100419 (2019).

24. F. Altaf, M. B. K. Niazi, Z. Jahan, T. Ahmad, M. A. Akram, A. Safdar, M. S. Butt, T. Noor, and F. Sher, J. Polym. Environ. (2020).

25. K. Pramod, C. V. Suneesh, S. Shanavas, S. H. Ansari, and J. Ali, J. Anal. Sci. Technol. 6, 34 (2015).

26. M. Rafiq, T. Hussain, S. Abid, A. Nazir, and R. Masood, Mater. Res. Express 5, 035007 (2018).

27. J. D. W. Rodríguez, S. Peyron, P. Rigou, and P. Chalier, PLoS One 13, e0207401 (2018).

28. L. Qi, Z. Xu, X. Jiang, C. Hu, and X. Zou, Carbohydr. Res. 339, 2693 (2004).

29. A. T. Hang, B. Tae, and J. S. Park, Carbohydr. Polym. 82, 472 (2010).

30. M. Anand, P. Sathyapriya, M. Maruthupandy, and A. Hameedha Beevi, Front. Lab. Med. 2, 72 (2018). 
31. A. L. HOLCK, M. K. PETTERSEN, M. H. MOEN, and O. SøRHEIM, J. Food Prot. 77, 1133 (2014).

32. S. Mathew, S. S, J. Mathew, and R. E.K., Food Packag. Shelf Life 19, 155 (2019).

33. A. Cano, M. Cháfer, A. Chiralt, and C. González-Martínez, Foods 5, 3 (2015).

34. Å. Nyflött, Ç. Meriçer, M. Minelli, E. Moons, L. Järnström, M. Lestelius, and M. G. Baschetti, J. Coatings Technol. Res. 14, 1345 (2017).

35. J. M. Lagaron and E. Núñez, J. Plast. Film Sheeting 28, 79 (2012).

36. G. Kavoosi, B. Nateghpoor, S. M. M. Dadfar, and S. M. A. Dadfar, J. Appl. Polym. Sci. 131, n/a (2014).

37. T. M. P. Ngo, T. H. Nguyen, T. M. Q. Dang, T. X. Tran, and P. Rachtanapun, Int. J. Mol. Sci. 21, 2224 (2020).

38. C. Rolfe and H. Daryaei, in (2020), pp. 3-24.

39. V. Gravel, C. Martinez, H. Antoun, and R. J. Tweddell, Biocontrol 50, 771 (2005).

40. H. S. Al-Hussini, A. Y. Al-Rawahi, A. A. Al-Marhoon, S. A. Al-Abri, I. H. Al-Mahmooli, A. M. Al-Sadi, and R. Velazhahan, J. Plant Pathol. 101, 315 (2019).

41. G. L. Vanti, S. Masaphy, M. Kurjogi, S. Chakrasali, and V. B. Nargund, Int. J. Biol. Macromol. 156, 1387 (2020).

42. K. Chaieb, H. Hajlaoui, T. Zmantar, A. Ben Kahla-Nakbi, M. Rouabhia, K. Mahdouani, and A. Bakhrouf, Phyther. Res. 21, 501 (2007).

43. Y.-N. Ma, F.-R. Xu, C.-J. Chen, Q.-Q. Li, M.-Z. Wang, Y.-X. Cheng, and X. Dong, Ind. Crops Prod. 133,185 (2019).

44. A. G. Luque-Alcaraz, M. O. Cortez-Rocha, C. A. Velázquez-Contreras, A. L. Acosta-Silva, H. del C. Santacruz-Ortega, A. Burgos-Hernández, W. M. Argüelles-Monal, and M. Plascencia-Jatomea, J. Nanomater. 2016, 1 (2016).

\section{Figures}




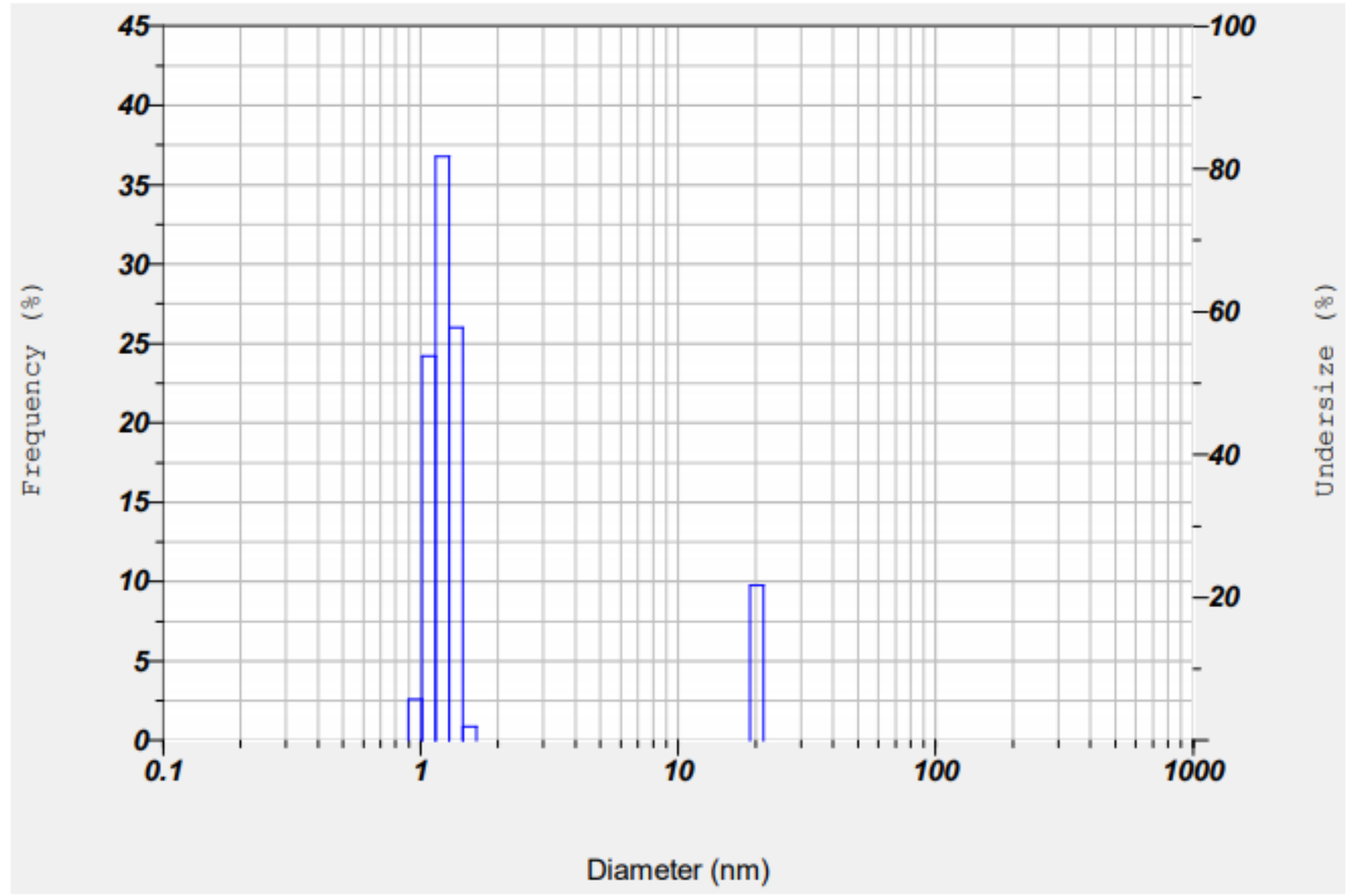

\section{Figure 1}

DLS analysis of the prepared chitosan nanoparticles indicating the mean size as $3.1 \mathrm{~nm}$ 

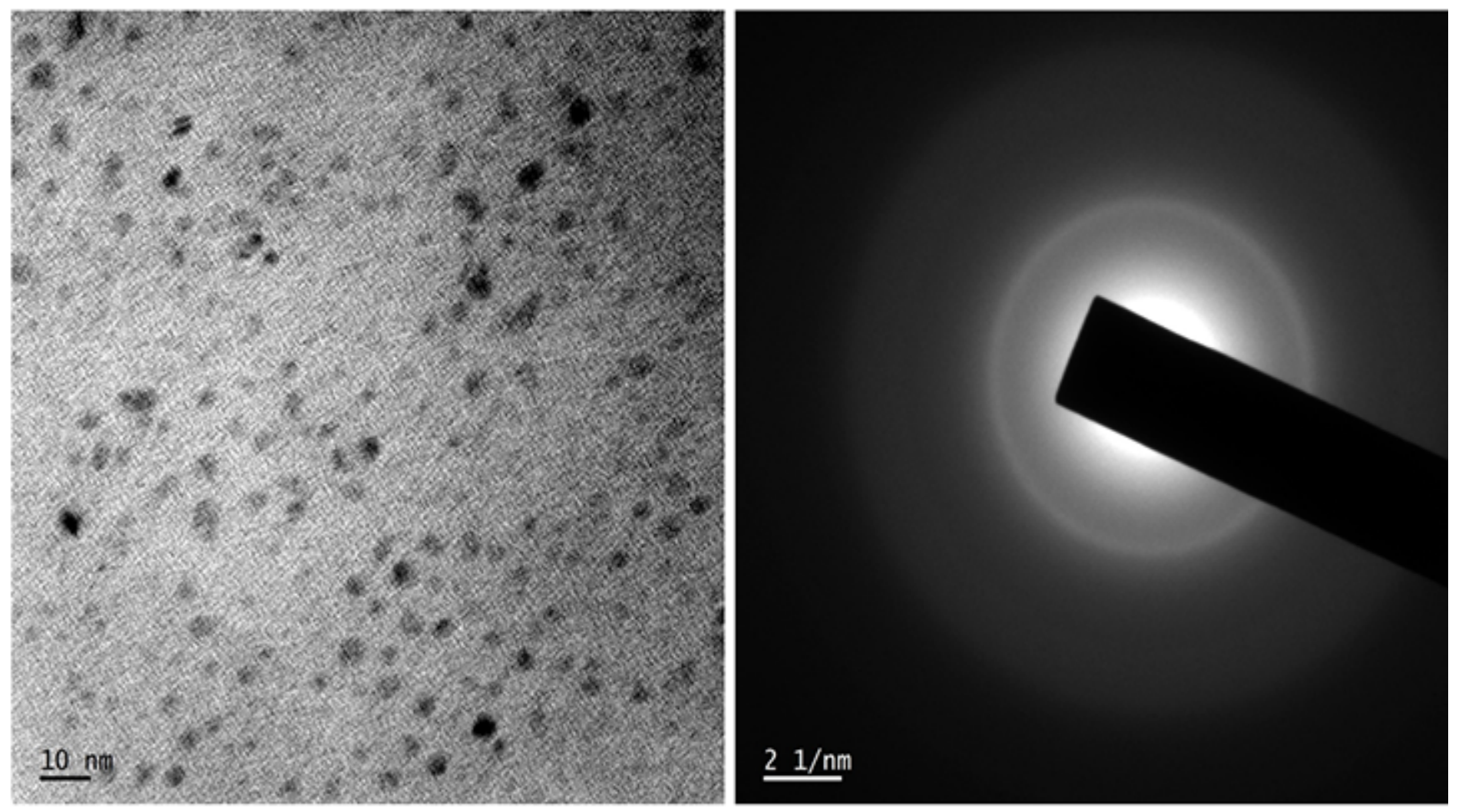

Figure 2

TEM image of prepared chitosan nanoparticle indicating spherical structure. 


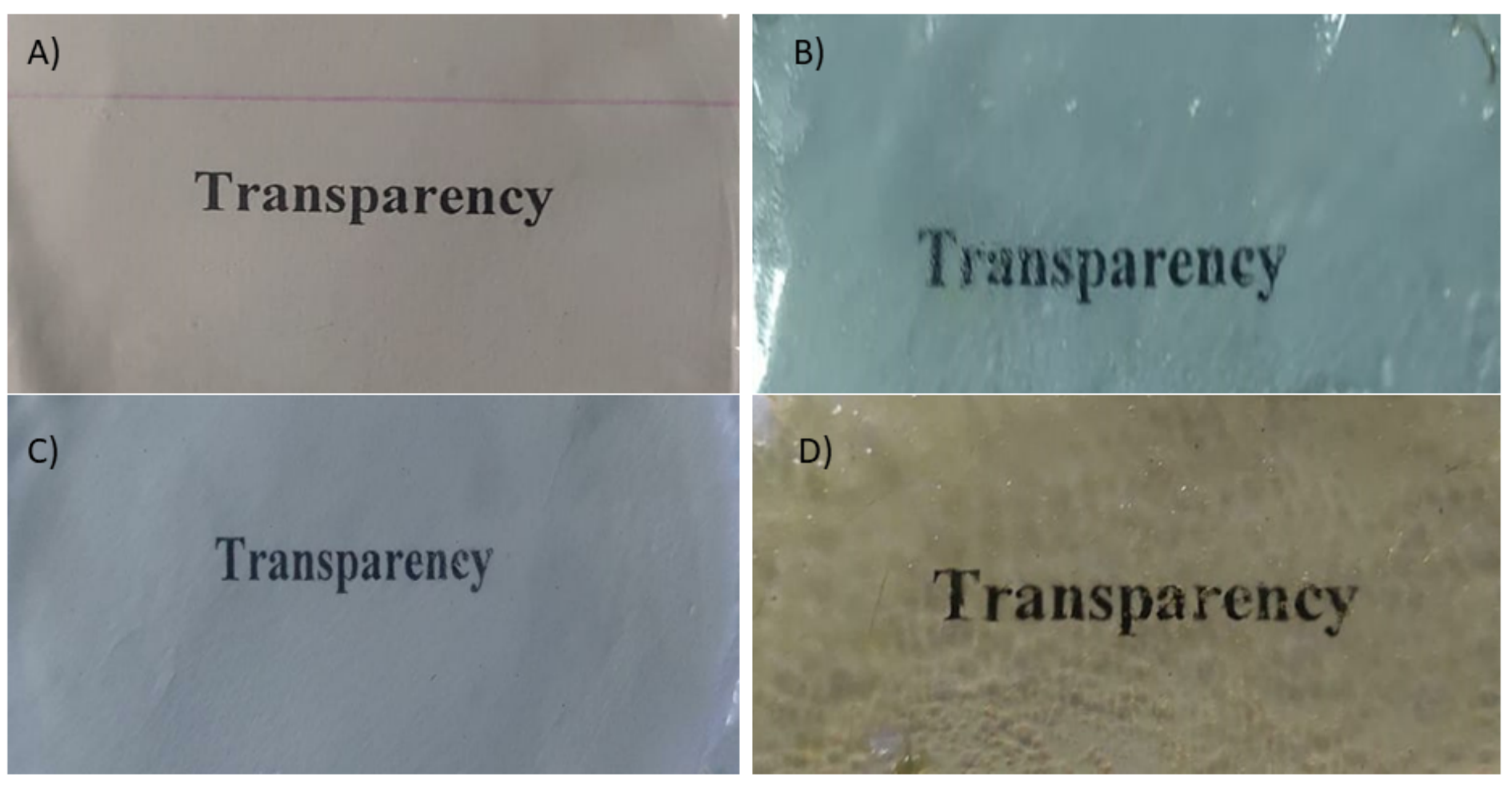

Figure 3

Transparency of the prepared bionanocomposites A)PVA, B) PCO, C) PCS and D) PCSCO 


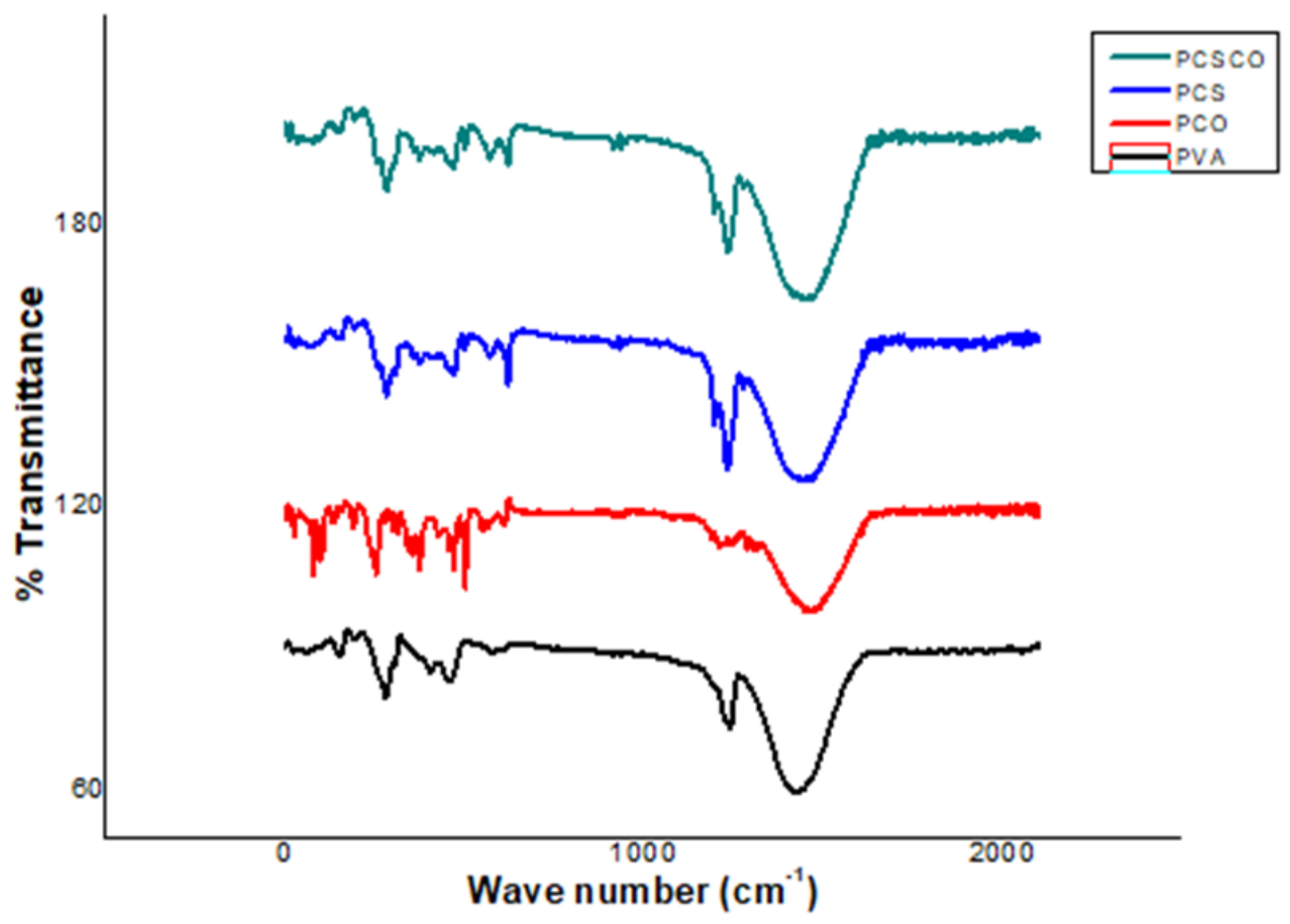

Figure 4

FTIR analysis of the prepared bionanocomposites PVA, PCO, PCS, PCSCO 


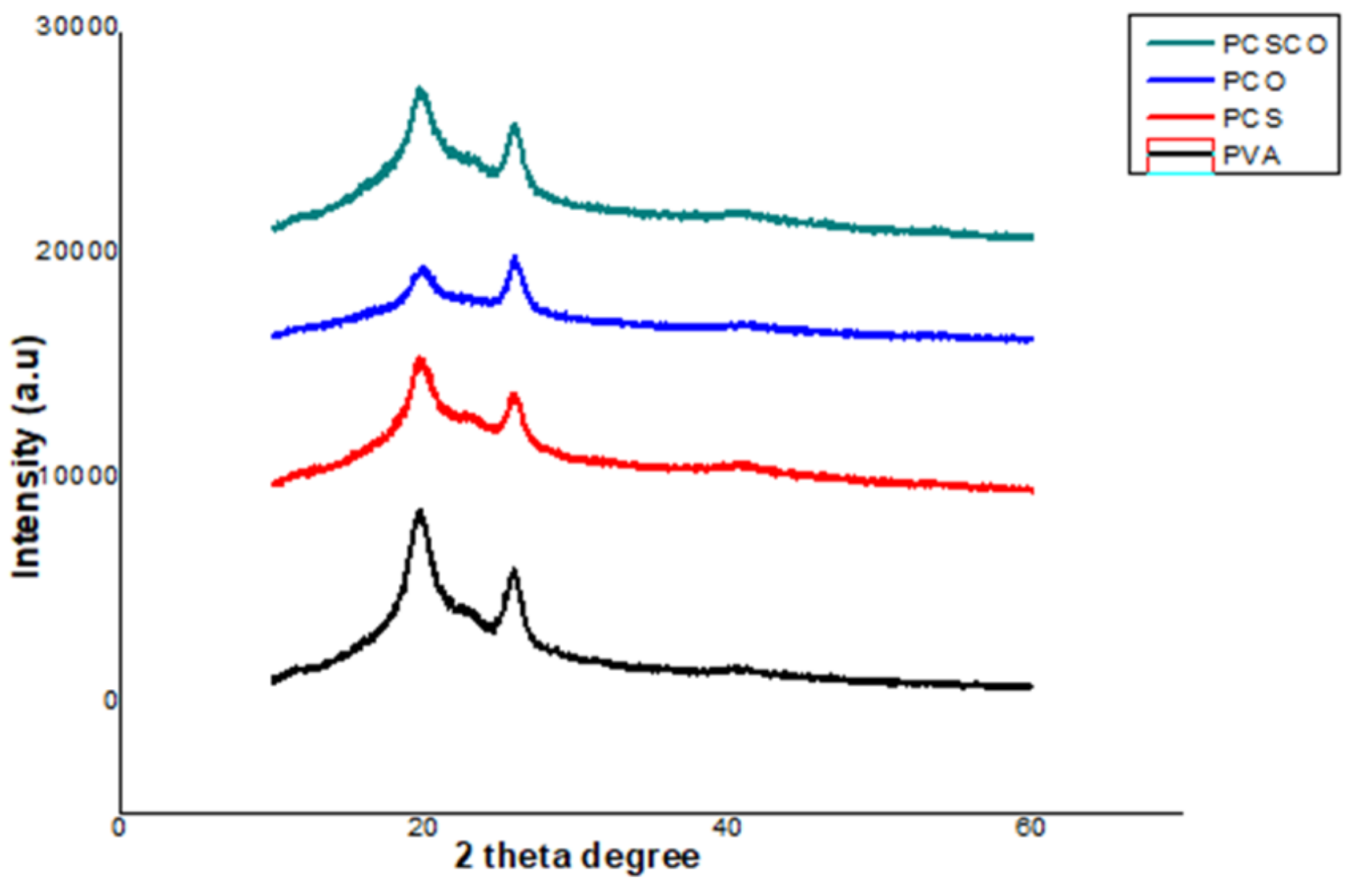

Figure 5

XRD analysis of the prepared bionanocomposites PVA, PCO, PCS, PCSCO 


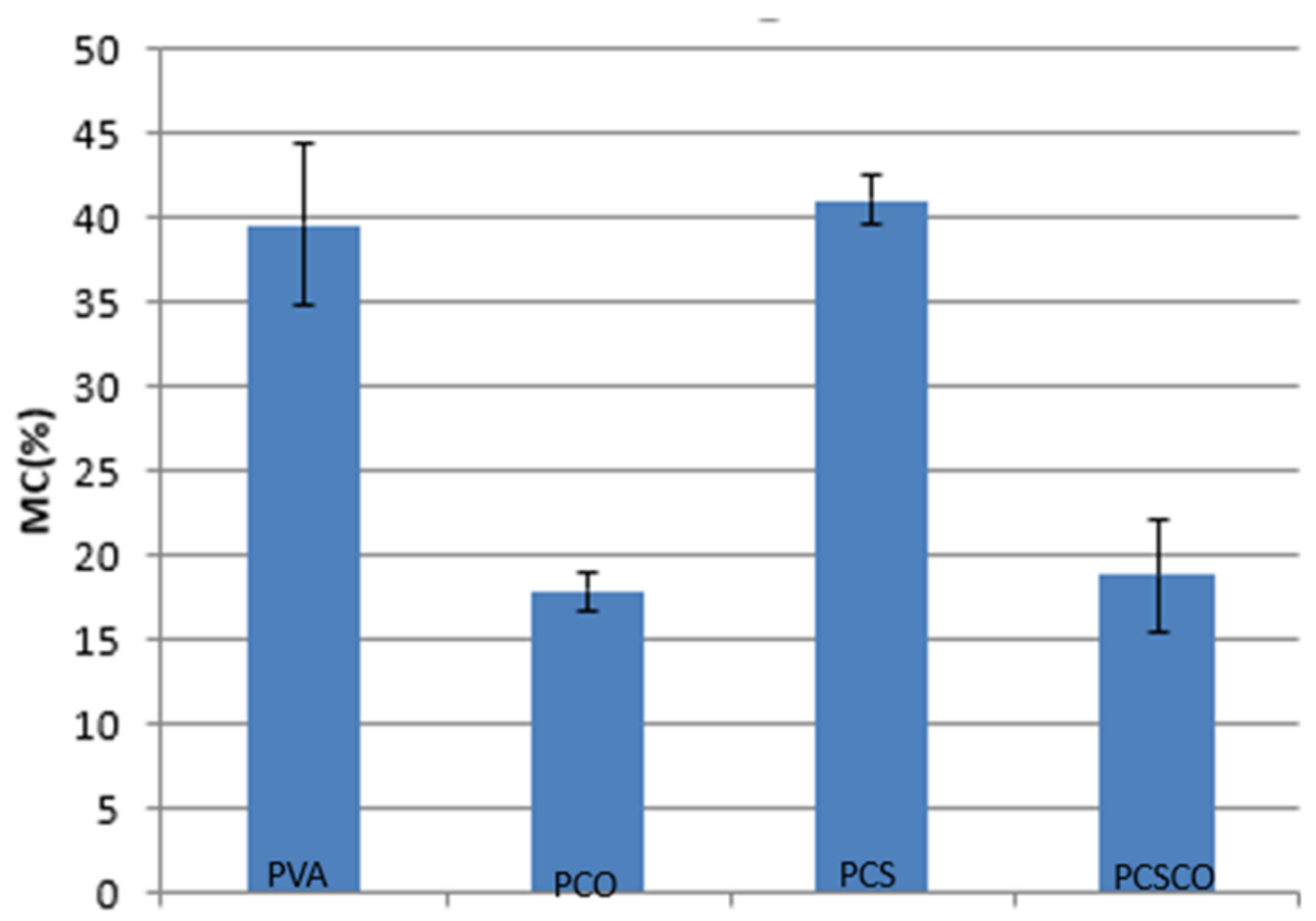

Figure 6

Moisture content of the prepared bionanocomposites PVA, PCO, PCS, PCSCO 


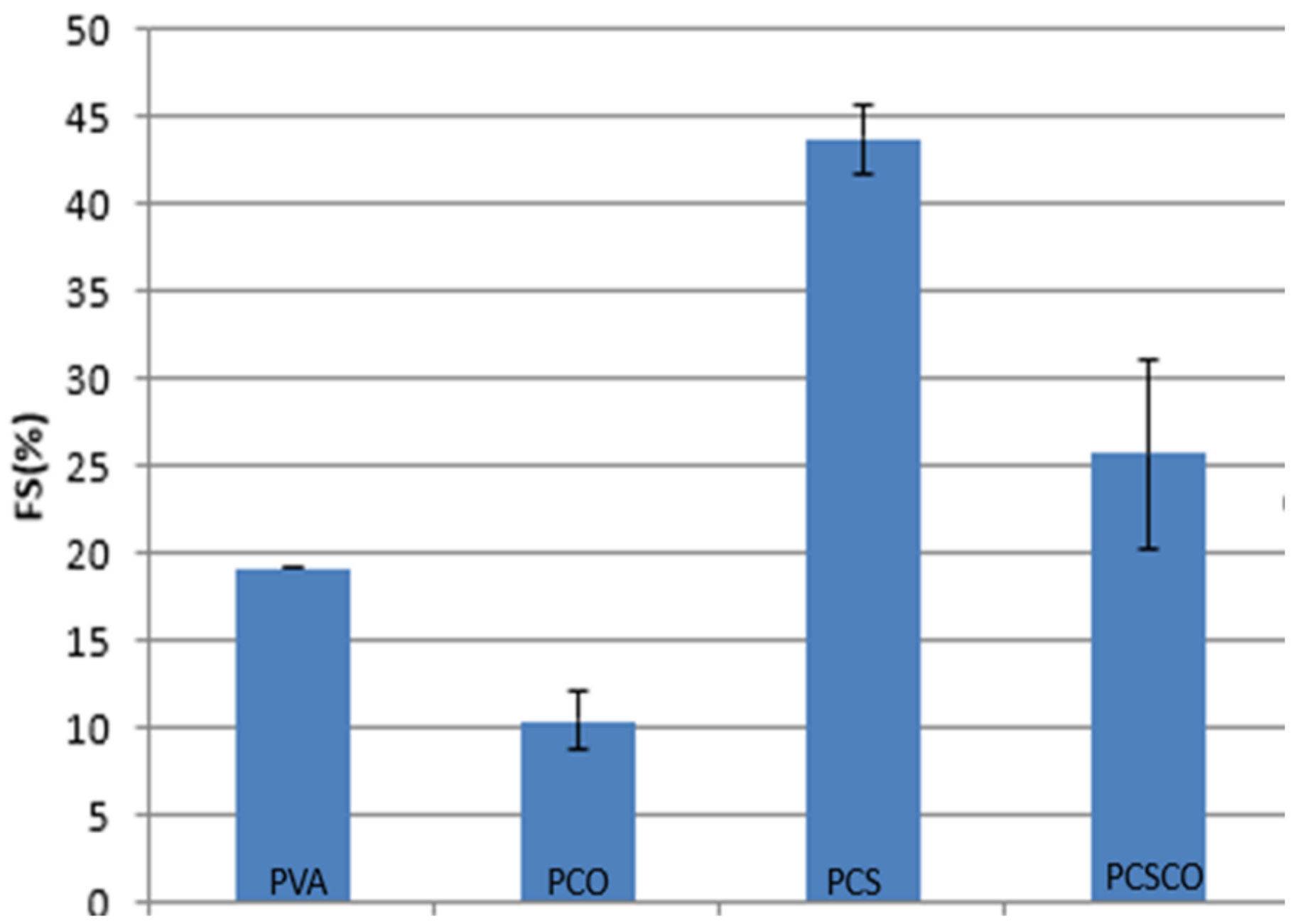

Figure 7

Film solubility of the prepared bionanocomposites PVA, PCO, PCS, PCSCO 

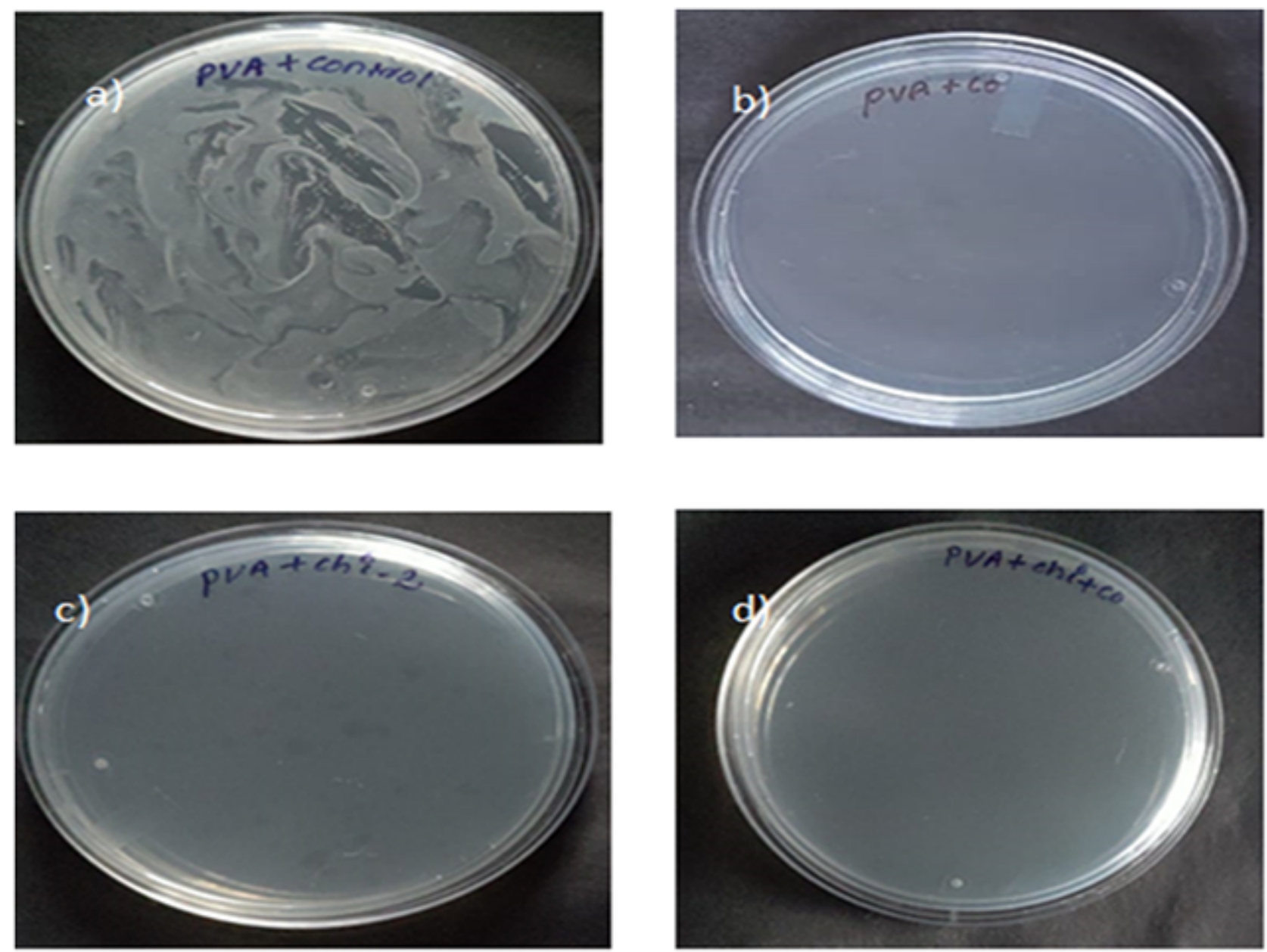

Figure 8

microbial barrier property of the prepared bionanocomposite films A) PVA, B) PCO, C) PCS, D)PCSCO 


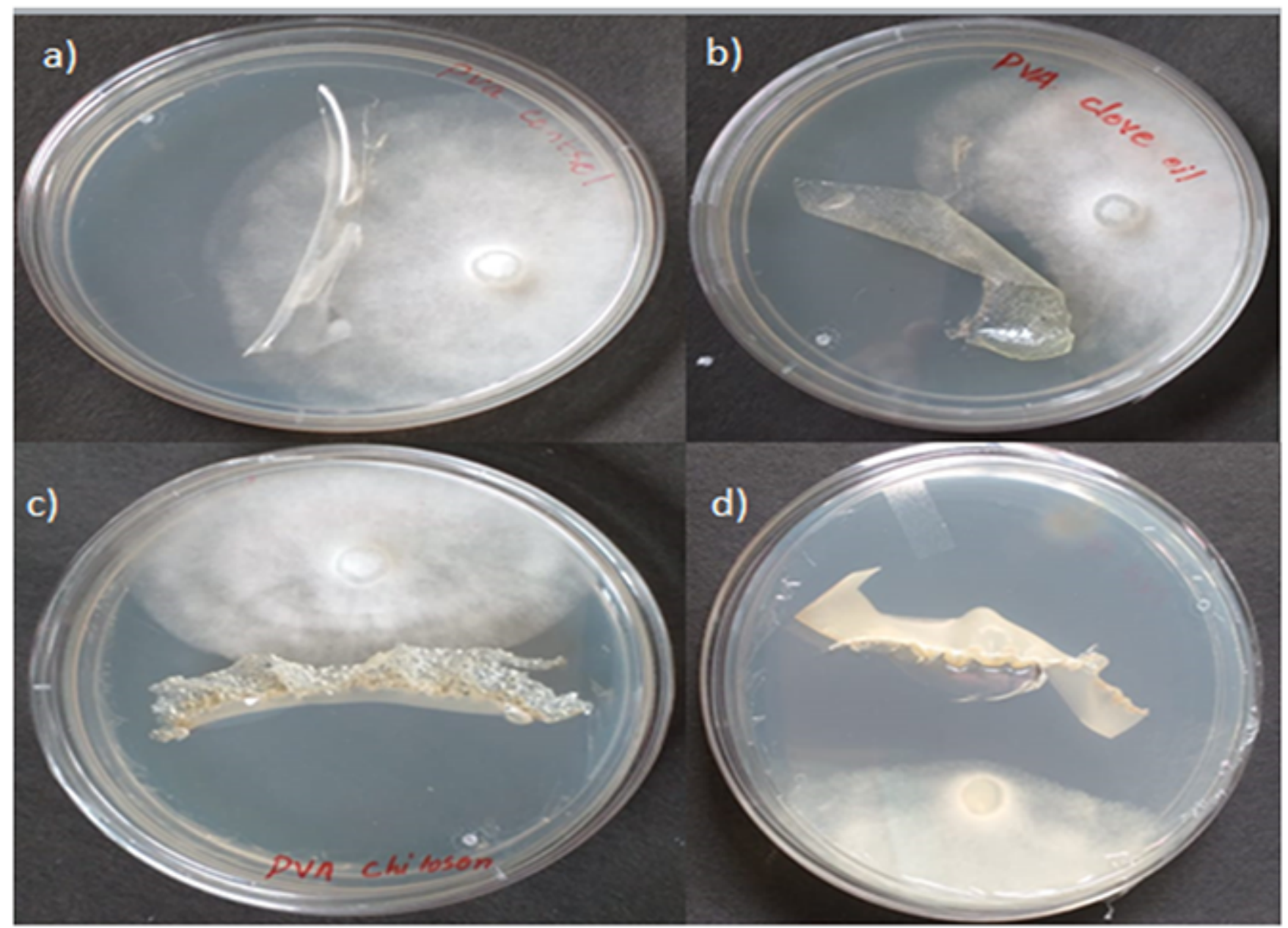

Figure 9

antifungal property of the prepared bionanocomposites A) PVA, B) PCO, C) PCS, D) PCSCO 